\title{
Finite Element Model of Cornea Deformation
}

\author{
Jessica R. Crouch ${ }^{1}$, John C. Merriam² ${ }^{2}$, and Earl R. Crouch III ${ }^{3}$ \\ 1 Computer Science Dept., Old Dominion University, VA, USA \\ jrcrouch@odu.edu \\ 2 Department of Ophthalmology, Columbia, New York, NY, USA \\ 3 Department of Ophthalmology, Eastern Virginia Medical School, Norfolk, VA, USA
}

\begin{abstract}
Cornea surgeons have observed that changes in cornea curvature can follow cataract surgery and cause astigmatism. The placement of surgical incisions has been shown to influence these curvature changes. Though empirical data has been collected about this phenomenon, a biomechanical model has not been employed in predicting post-surgical outcomes. This work implemented an incised finite element model of the eye to investigate factors influencing corneal shape after surgery. In particular, the effects of eye muscle forces and intra-ocular pressure were simulated. Cornea shape change was computed via finite element analysis, and the resulting change in cornea curvature was measured by fitting quadratic curves to the horizontal and vertical meridians of the cornea. Results suggest that these two sources of deforming force counteract each other and contribute to astigmatism in perpendicular directions.
\end{abstract}

\section{Introduction}

A finite element model of the eye was developed to investigate whether such a model might be helpful in predicting changes in the corneal curvature after cataract surgery. Cornea surgeons have observed that changes in cornea curvature often follow cataract surgery and alter pre-operative astigmatism. Further, they have found that the orientation of the astigmatism is related to the position of the surgical incision(s). For patients with pre-existing astigmatism, the astigmatism can be reduced or increased, depending on incision placement. Cornea surgeons have produced tables based on empirical observations of surgical results that allow them to predict the cornea curvature change that will result from a given incision [1]. However, a clear mechanical understanding of the causes of the observed changes does not exist.

Related work includes a study by Pinsky and Datye 2 that constructed a finite element model of the eye to examine the effects of radial keratotomy. Partial thickness corneal incisions were modeled on one quarter of the cornea; horizontal and vertical corneal symmetry was assumed. The work presented here expands on this earlier work by modeling the full cornea and the rest of the globe without making assumptions about symmetry that prevent the consideration of irregularities such as astigmatism. Another difference is the placement and depth of the modeled incision. This work models an incision along the periphery of the 
cornea that might be used for cataract or anterior segment surgery instead of the radial incisions used in radial keratotomy.

Other models of radial keratotomy have been developed by Vito, et al [3], Wray, et al 4], and Sawusch, et al [5]. Related work also includes a model of the phototherapeutic keratectomy procedure by Katsube, et al [6] and a simulation of eye trauma using a finite element model that was constructed by Uchio, et al [7.

Investigations into the material properties of the cornea provide a basis for the development of biomechanical models. Such work includes an analysis of cornea strain distribution by Shin, et al 8 and a micro-structural analysis of the cornea by Johnson 9 .

The construction of the finite element mode used in this work is described in section 2, along with the simulated incision and application of deforming forces. Model deformation results are described in section 3 and discussed in light of the available patient data. Finally, section 4 presents conclusions and planned future work.

\section{Finite Element Model}

The finite element eye model in this study was designed to focus on the structural integrity of the cornea and sclera, the structures that form the outer shell of the eye. Initially, a geometric eye model was developed based on the female Visible Human image set, using anatomical image slices with $0.33 \mathrm{~mm}$ thickness. However, experience showed that the image resolution and clarity was not sufficient to adequately capture the small, detailed structures of the eye. A smoother, more appropriate model was subsequently developed using an analytically generated eye model based on the known geometry of the eye. This type of model has the further advantage of providing automatic generation of eye models to represent patients with a variety of eye and cornea geometry.

The model generation algorithm accepts as input the radii of curvature for the cornea and the larger eye globe, as well as the number and thickness of the layers that form the outer shell of the eye. The algorithm produces as output a finite element mesh composed of quadratic hexahedral elements. Quadratic hexahedra provide excellent representation of the eye's smooth curved surface geometry with a relatively small number of mesh nodes and elements. Each quadratic hexahedral elements had 27 nodes, as shown in Fig. 1. The eye mesh



Fig. 1. Illustration of the structure of a quadratic hexahedral element. Each edge interpolates 3 nodes, resulting in a quadratic curve. The midpoint of each face and the center of the element also have nodes that support quadratic interpolation across all three spatial dimensions. 
shown in Fig. 2 contains only 6804 nodes and 812 elements. Hexahedral elements also provide excellent numerical properties for finite element analysis [10].

The eye model is generated using two superimposed spherical coordinate systems. The model shown in Fig. 2 has realistic eye geometry, with a globe radius of $11.5 \mathrm{~mm}$ and a cornea radius of $7.8 \mathrm{~mm}$. A linear elastic material model was used for the entire eye, but the stiffness and thickness of the different layers of the cornea varied. For the model in Fig. 2, the corneal layers were represented as shown in Table 1.

Mesh elements were created in layers to form concentric spherical shells. Each node was provided with both spherical coordinates $(\theta, \phi, r)$ and Cartesian coordinates $(\mathrm{x}, \mathrm{y}, \mathrm{z})$. A custom meshing pattern was developed to connect the cornea mesh and the globe mesh so that the eye model consisted of one continuous finite element mesh. For this model, the interior of the eye was assumed to have homogeneous material properties.

A linear elastic material model was applied to the eye model. The thick stroma layers of the cornea are composed predominantly of collagen and water. These layers account for much of the cornea's strength, and were assigned a Young's modulus $160 \mathrm{kPa}$, consistent with published stiffness values [11, and a Poisson's ratio of 0.49 to indicate near incompressibility. The shell of the globe was assigned the same material properties, while the interior of the eye and thinner layers of the cornea were assigned the same Poisson's ratio but were
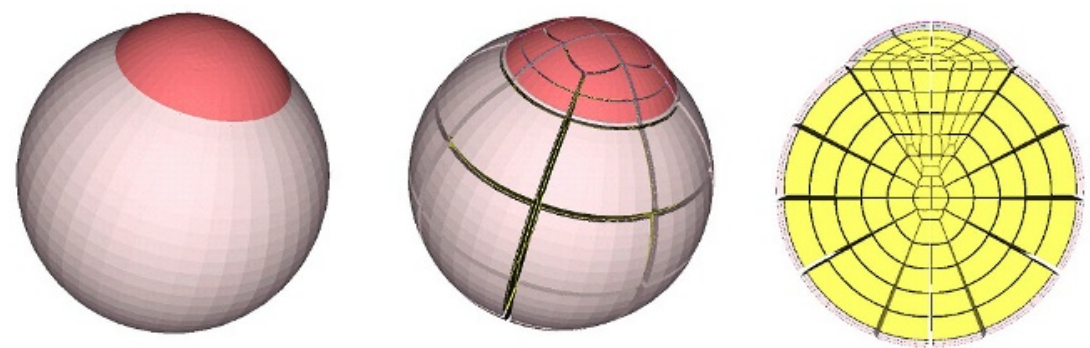

Fig. 2. Left: Exterior view of an eye model. Center: View showing divisions between the quadratic hexahedral elements that compose the finite element mesh. Right: Interior view of the structure of the volumetric mesh.

Table 1. Eye model layers and thicknesses

\begin{tabular}{|l|c|}
\hline Name & Thickness $(\mathrm{mm})$ \\
\hline Epithelium & 0.05 \\
\hline Bowman's membrane & 0.012 \\
\hline Stroma (anterior) & 0.25 \\
\hline Stroma (posterior) & 0.25 \\
\hline Descemet's membrane & 0.01 \\
\hline Endothelium & 0.005 \\
\hline
\end{tabular}




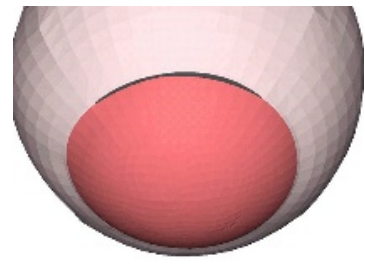

Fig. 3. To model an incision along the top edge of the cornea, connections between adjacent elements were removed through all of the corneal layers. Connections between the elements that compose the homogeneous interior of the eye were left intact. The black curve along the top corneal border indicates the placement of the cut.

given a Young's modulus an order of magnitude smaller. This choice of material properties was designed to capture the difference between the relatively tough outer shell of the eye and the interior of the eye that is filled with a much softer vitreous material.

To examine the effects of surgical incisions, a cut in the initial eye mesh was made, as shown in Fig. 3. The $9.5 \mathrm{~mm}$ incision was made along the top border of the cornea and represents the incision made in an extra-capsular cataract extraction procedure. The incision alone does not cause post-operative astigmatism, but forces acting on an incised cornea cause it to deform differently than an intact cornea would. Therefore, two types of forces were defined to simulate the stresses to which corneas are ordinarily subjected.

The first set of forces represented intra-ocular pressure. In this case, a uniform force was applied across the cornea in the direction normal to the cornea surface at each point. The normal range for intra-ocular pressure is $10-21 \mathrm{~mm} \mathrm{Hg}$. For this experiment a pressure of $10 \mathrm{~mm} \mathrm{Hg}$ was applied, which is equal to $1.33 \mathrm{kPa}$ distributed over the surface area of the cornea.

The second set of forces were muscular. The superior, inferior, lateral, and medial recti eye muscles are the strongest muscles attached to the eye, and these were approximated by force vectors applied tangentially at the locations where those muscles insert on the globe. The applied muscle forces had three times the
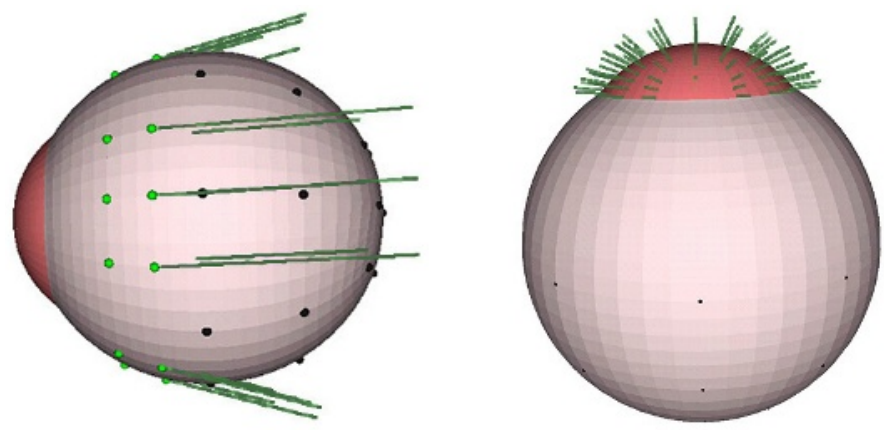

Fig. 4. Illustration of two sets of forces applied to the eye. For both sets of boundary conditions, nodes on the posterior portion of the eye remained fixed. These are marked with black dots. Left: Eye model with muscle forces applied tangential to the globe. Right: Eye model with intra-ocular pressure forces applied normal to the cornea. 
magnitude of the intra-ocular pressure, but were applied to a smaller area. The force from each muscle was applied to a narrow patch located approximately $6.5 \mathrm{~mm}$ from the edge of the cornea. Fig. 4 illustrates both the muscle and intra-ocular force vectors. To maintain stability and prevent model translation or rotation under the application of these forces, the nodes on the posterior surface of the globe were assigned fixed positions.

The application of forces to the incised cornea model produced a deformed corneal surface, but further analysis was required to quantify the shape change. The shape change was analyzed by performing a least-squares fit of quadratic polynomial curves to the mesh nodes lying along the horizontal and vertical meridians of the deformed corneas. The radius of curvature of the quadratic curves was computed at the center point of the cornea, and then the radius of curvature was converted to diopters, a measure of refractive power. This allowed direct comparison between the model results and published patient data. Results are presented in the following section.

\section{Results}

Visualization of the height maps of the original and deformed corneas produced by the two sets of boundary conditions is provided in Fig. 5. Corresponding numerical results are shown in Table 2.

The results show that for an incision along the top border of the cornea model, muscle forces led to stretching and flattening of the cornea along the horizontal meridian. Forces from intra-ocular pressure led to stretching and flattening along the vertical meridian. This indicates that the two types of forces tend to counteract each other in terms of causing astigmatism.

Merriam showed that for the type of cornea incision modeled in this experiment, one month after surgery patients had an average of +1.6 diopters change in the vertical meridian (steepening) and -1.4 diopters change in the horizontal
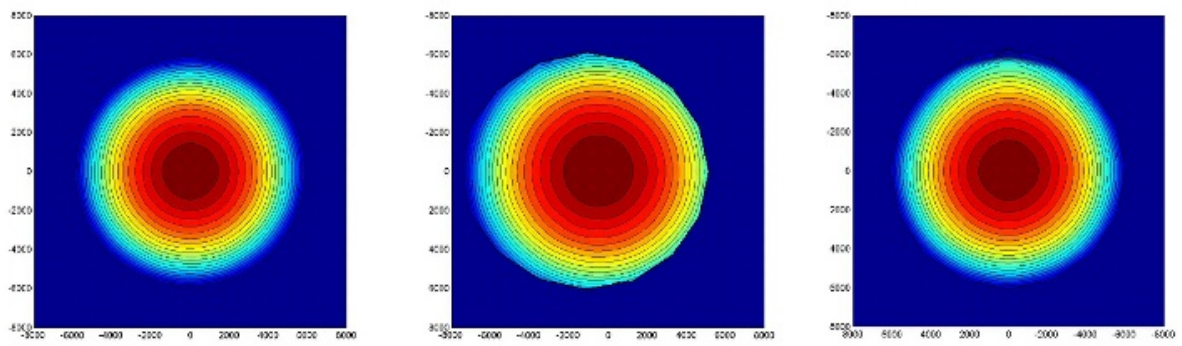

Fig. 5. Left: Contours of original, spherical cornea's height map Center: Contours of cornea model deformed by muscle forces, showing evidence of flattening in the horizontal direction Right: Contours of cornea model deformed by intra-ocular pressure, showing evidence of flattening in the vertical direction, but less pronounced than the flattening caused the muscle forces. 
Table 2. Numerical results for the finite element model's cornea curvature across the horizontal and vertical meridians, given muscle force boundary conditions and intraocular pressure boundary conditions

\begin{tabular}{|l|c|c|c|c|}
\hline & $\begin{array}{c}\text { Vertical Axis } \\
\text { Radius of } \\
\text { Curvature }(\mathrm{mm})\end{array}$ & $\begin{array}{c}\text { Vertical Axis } \\
\text { Diopters }\end{array}$ & $\begin{array}{c}\text { Horizontal Axis } \\
\text { Radius of } \\
\text { Curvature }(\mathrm{mm})\end{array}$ & $\begin{array}{c}\text { Horizontal Axis } \\
\text { Diopters }\end{array}$ \\
\hline Original Cornea & 7.80 & 43.27 & 7.80 & 43.27 \\
\hline $\begin{array}{l}\text { Deformed by } \\
\text { muscle forces }\end{array}$ & 7.64 & 44.18 & 7.72 & 43.72 \\
\hline $\begin{array}{l}\text { Deformed by } \\
\text { intra-ocular } \\
\text { pressure }\end{array}$ & 7.89 & 42.76 & 7.59 & 44.47 \\
\hline
\end{tabular}

meridian (flattening). However, five years after surgery patients exhibited astigmatism in the reverse direction, with an average of -0.7 diopters change in the vertical meridian and +0.6 diopters change in the horizontal meridian compared to their pre-operative condition [1. This is likely because the vision change in first weeks after surgery is heavily influenced by tissue swelling and the healing response, whereas the long term vision change may be due to permanent structural changes in the cornea.

The finite element model results indicate that the long-term astigmatism observed in the patient population is consistent with the model deformation caused by forces from intra-ocular pressure. However this preliminary result requires further investigation, as not all of the factors influencing the cornea shape were represented in the initial finite element model. In particular, the effects of swelling and healing may influence the final visual result. In the healing process, scar tissue is formed that has different mechanical properties than normal cornea tissue. This scar tissue begins to form while the eye is still swollen from the surgical trauma. Therefore the final, healed geometry of the eye may depend on a patient's degree of swelling, rate of healing, and scar formation process as well as the forces exerted by intra-ocular pressure and eye muscles.

\section{Conclusions and Future Work}

In conclusion, this work has shown that the long-term astigmatism observed in the patient population is qualitatively similar to the model deformation caused by forces from intra-ocular pressure. However, extensive future work is needed to produce and validate a biomechanical model that could be used to help predict surgical outcomes.

Future work may expand on the current work in the following ways:

- Apply an oriented fiber tissue model to cornea.

The corneal stroma is known to be composed of oriented bundles of 
collagen [12. The direction of collagen fibrils at a given point on the cornea is related to that point's distance from the center of the cornea and angle from the horizontal and vertical axes. A finite element oriented fiber material model requires that each element possess a vector that defines the direction of the fibers. Although an isotropic material model was assumed for this initial eye model, the use of spherical coordinate systems in the mesh construction will facilitate the future implementation of an oriented fiber material model for the cornea.

- Augment the finite element model with a time-dependent simulation of the healing response.

By locally varying the cornea's material properties over time to account for swelling and scar formation, and then running the model over a number of simulated months, the impact of the healing process could be examined. A time-dependent simulation would also allow the influence of constant intraocular pressure versus the intermittent application of muscle forces to be investigated.

- Experiment with individualized eye models.

By generating a variety of eye models with different corneal thickness, different degrees and orientations of pre-existing astigmatism, different levels of intra-ocular pressure, and different levels of muscle strength, the model generated deformations could be compared to existing data on patient surgical outcomes. This would assist in the development and validation of an individualizable, predictive cataract surgery model. Additionally, the effect of incision placement and size can be examined for the different eye models to see if surgeons' ability to reduce pre-existing astigmatism with carefully placed incisions can be accurately replicated by the model.

\section{References}

1. Merriam, J., Zheng, L., Merriam, J., Zaider, M.: The effect of incisions for cataract on corneal curvature. Ophthalmology 110 (2003) 1807-1813

2. Pinsky, P., Datye, D.: A microstructurally based finite element model of the incised human cornea. Journal of Biomechanics 24 (1991) 907-992

3. Vito, R.P., Shin, T.J., McCarey, B.E.: A mechanical model of the cornea: the effects of physiological and surgical factors on radial keratotomy surgery. Refract Corneal Surg 5 (1989) 82-88

4. Wray, W., Best, E., Cheng, L.: A mechanical model for radial keratotomy: toward a predictive capability. Journal of Biomechanical Engineering 116 (1994) 56-61

5. Sawusch, M., McDonnell, P.: Computer modeling of wound gape following radial keratotomy. Refractive Corneal Surgery 8 (1992) 143-145

6. Katsube, N., Wang, R., Okuma, E., Roberts, C.: Biomechanical response of the cornea to phototherapeutic keratectomy when treated as a fluid-filled porous material. Journal of Refractive Surgery 18 (2002) 593-597

7. Uchio, E., Ohno, S., Kudoh, J., Aoki, K., Kisielewicz, L.: Simulation model of an eyeball based on finite element analysis on a supercomputer. British Journal of Ophthalmology 83 (1999) 1106-1111 
8. Shin, T., Vito, R., Johnson, L., McCarey, B.: The distribution of strain in the human cornea. Journal of Biomechanics 30 (1997) 497-503

9. Johnson, L.: The Mechanical and Microstructural Analysis of the Human Cornea. Dissertation, School of Mechanical Engineering, Georgia Institute of Technology (1998)

10. Benzley, S.E., Perry, E., Merkley, K., Clark, B., Sjaardama, G.: A comparison of all hexagonal and all tetrahedral finite element meshes for elastic and elasto-plastic analysis. Proceedings, 4th International Meshing Roundtable (1995) 179-191

11. Forster, W., Kasprzak, H., von Bally, G.: Measurement of elastic modulus of the central bovine cornea by means of holographic interferometry. Part II. Results. Optom Vis Science 71 (1994) 27-32

12. Hollman, K., Emelianov, S., Neiss, J., Jotyan, G., Spooner, G., Juhasz, T., Kurtz, R., O'Donnell, M.: Strain imaging of corneal tissue with an ultrasound elasticity microscope. Cornea 21 (2002) 68-73

13. Aghamohammadzadeh, H., Newton, R., Meek, K.: X-ray scattering used to map the preferred collagen orientation in the human cornea and limbus. Structure (Camb) 12 (2004) 249-256

14. Hjortdal, J.: Regional elastic performance of the human cornea. Journal of Biomechanics 29 (1996) 831-942

15. Sagar, M., Bullivant, D., Mallinson, G., Hunter, P.: A virtual environment and model of the eye for surgical simulation. International Conference on Computer Graphics and Interactive Techniques, Proceedings of the 21st annual conference on Computer graphics and interactive techniques (1994) 205-212 